\title{
PRODUÇÃO DE MICROCÁPSULAS DO EXTRATO SUPERCRÍTICO DE JABUTICABA (PLINIA TRUNCIFLORA) ATRAVÉS DA ATOMIZAÇÃO EM SPRAY DRYER
}

\author{
BOHN $^{1}$, F. KUHN 2 , O. TOMAZELLI Jr ${ }^{3}$, J. SCAPINELLO ${ }^{1}$, J. DAL MAGRO² \\ ${ }^{1}$ Universidade Comunitária da Região de Chapecó - Unochapecó, laboratório de Produtos \\ Naturais \\ ²Universidade Comunitária da Região de Chapecó - Unochapecó, Programa de Pós-Graduação \\ em Ciências Ambientais \\ ${ }^{3}$ Empresa de Pesquisa Agropecuária e Extensão Rural de Santa Catarina - EPAGRI \\ E-mail para contato: alinebohn@unochapeco.edu.br
}

RESUMO - Nos últimos anos, a jabuticaba tem sido alvo de inúmeras pesquisas, devido à sua elevada atividade antioxidante, podendo seu uso ser promissor nos setores alimentício e farmacêutico. Para prevenir a perda de compostos ativos, objetivou-se no presente trabalho microencapsular o extrato de jabuticaba, obtido através da extração com dióxido de carbono supercrítico. A microencapsulação foi realizada através da atomização do extrato em spray dryer, com a utilização da maltodextrina como material de parede das microcápsulas. $\mathrm{O}$ teor de compostos fenólicos totais e antocianinas monoméricas presentes no extrato bruto "livre" e microencapsulado não diferiu significativamente $(\mathrm{p}<0,05)$, bem como o potencial antioxidante medido através da captura do radical DPPH $(85,11 \pm 0,62 \%)$. A atomização mostrou-se eficiente e vantajosa na microencapsulação do extrato supercrítico de jabuticaba (Plinia trunciflora) uma vez que não ocasionou perdas dos compostos bioativos durante o processo.

Palavras - chave: Plinia trunciflora, microencapsulamento, spray dryer.

\section{INTRODUÇÃO}

Muitos estudos têm mostrado que a jabuticaba possui notável atividade antioxidante, devido ao teor significativo de compostos fenólicos e principalmente devido à presença de antocianinas Einbond et al. (2004); Reynertson et al. (2005); Reynertson et al. (2008). Estas, por sua vez, têm atraído à atenção da comunidade científica devido as suas atividades biológicas. Antocianinas de diversas de plantas, dentre elas a jabuticaba, têm sido testadas, uma vez que podem ser ativas na redução do estresse oxidativo e na prevenção de muitas doenças (BRITO et al., 2007; DAI et al., 2009; LEITE-LEGATTI et al., 2012).

No entanto, o uso das antocianinas na área alimentícia e farmacêutica tem demonstrado ser um grande desafio tecnológico, uma vez que estes compostos possuem pouca estabilidade a condições ambientais durante o processamento, armazenamento e também durante o consumo. As antocianinas são susceptíveis a degradação por meio de fatores tais como a presença de luz, de 
$\mathrm{pH}$ (principalmente $\mathrm{pH}$ superior a 7), temperaturas superiores a $60-80{ }^{\circ} \mathrm{C}$, entre outros fatores (Giusti e Wallace, 2009; Wesche-Ebeling e Argaiz-Jamet, 2002). Uma possível maneira de proteger eficazmente as antocianinas de fatores adversos, como descritos anteriormente, seria a utilização de técnicas de encapsulação como a microencapsulação por spray dryer.

A microencapsulação pode ser definida como uma tecnologia de empacotamento com finas coberturas poliméricas aplicáveis em sólidos, gotículas de líquidos ou material gasoso, formando pequenas partículas denominadas microcápsulas, que podem liberar seu conteúdo sob velocidade e condições específicas (TODD, 1970).

As microcápsulas têm a capacidade de modificar e melhorar a aparência e as propriedades de uma substância, tais como, reduzir a reatividade do material de núcleo com o ambiente, diminuir a velocidade de evaporação ou de transferência do material de núcleo para o meio, facilitar a manipulação do material encapsulado, promover a liberação controlada, mascarar sabor e odor desagradáveis, além de promover a diluição homogênea do material encapsulado em uma formulação (DUBEY et al., 2009; SHAHIDI e HAN, 1993; GHARSALLAOUI et al., 2007; SUAVE et al., 2006).

A atomização é a técnica mais comum e mais barata para a produção de produtos microencapsulados, pois os custos de produção são inferiores a maioria dos outros métodos. Comparado à liofilização, o custo da técnica utilizando spray dryer é 3 - 50 vezes mais barata (DESOBRY, et al., 1997).

Entre as tecnologias utilizadas para obtenção de extratos vegetais, a extração com fluido supercrítico apresenta algumas vantagens frente aos métodos convencionais de extração, como a obtenção de um extrato mais purificado devido à ausência de solventes orgânicos, menor geração de resíduos e até mesmo menores temperaturas de processamento. A extração com fluído supercrítico $\mathrm{CO}_{2}\left(\mathrm{SC}-\mathrm{CO}_{2}\right)$ passou a ser empregada na extração de metabólitos secundários de diferentes estruturas vegetais, se mostrando como uma técnica promissora para obtenção de extratos ricos em compostos naturais de interesse (NAHAR; SARKER, 2006).

Nesse contexto, o presente trabalho teve por objetivo microencapsular o extrato de jabuticaba (Plinia trunciflora) obtido através da extração com fluído supercrítico $\left(\mathrm{CO}_{2}\right)$ empregando a técnica de atomização por spray dryer. 


\section{9 a 22 de outubro de 2014 \\ Florianópolis/SC}

\section{MATERIAL E MÉTODOS}

\subsection{Elaboração do extrato dos frutos de jabuticaba}

Os frutos "in natura" de Plinia trunciflora foram coletados em setembro de 2012 na Linha Barra Grande, no município de Alpestre, Rio Grande do Sul $\left(27^{\circ} 10^{\prime} 56.82^{\prime S}\right.$ e 53 7'19.55"O, altitude de $224 \mathrm{~m})$.

A extração com $\mathrm{CO}_{2}$ supercrítico foi realizada nas condições de pressão e temperatura descritas por Lin et al. (1999). $60 \mathrm{~g}$ de frutos inteiros congelados de jabuticaba foram triturados até a obtenção de partículas com diâmetro inferior a $1 \mathrm{~mm}$. Em seguida, foram introduzidas no extrator supercrítico composto por uma bomba de pressurização (DLE 30 - Maxpro Technologies), um banho termostatizado (TE - 180 - Tecnal), compressor de ar e um cilindro de gás carbônico (Air liquide) e submetidas ao processo de extração com $\mathrm{CO}_{2}$ a 200 bar e $60{ }^{\circ} \mathrm{C}$ durante 2 horas. Após a extração, o extrato foi liofilizado e congelado até a realização das análises propostas.

\subsection{Elaboração das microcápsulas de jabuticaba}

Para a produção das microcápsulas, foram utilizados $25 \%$ de extrato seco, $30 \%$ de maltodextrina (ALDRICH) e água destilada. Essa solução permaneceu sob agitação constante por 2 horas a $500 \mathrm{rpm}$ em um agitador eletromagnético (Selecta - Agimatic). Em seguida, foi submetida ao processo de atomização em um spray dryer (Buchi B 290), com as seguintes condições de operação: temperatura de entrada $140{ }^{\circ} \mathrm{C}$; temperatura de saída $80{ }^{\circ} \mathrm{C}$; vazão do ar de secagem $438 \mathrm{~L} /$ hora; fluxo de alimentação $12 \mathrm{~mL} / \mathrm{mim}$.

\subsection{Polifenóis totais, antocianinas monoméricas e atividade antioxidante}

O conteúdo fenólico total foi determinado pelo método de Folin-Ciocaulteu, conforme metodologia proposta por Shahidi e Naczk (1995), com algumas modificações. As antocianinas monoméricas totais foram quantificadas de acordo com Giusti e Wrolstad (2001), no qual foram utilizados dois sistemas tampão: ácido clorídrico/cloreto de potássio de $\mathrm{pH}$ 1,0 $(0,025 \mathrm{M})$ e ácido acético/acetato de sódio de $\mathrm{pH}$ 4,5 (0,4 M). A atividade antioxidante foi avaliada através do monitoramento do radical DPPH, com base na metodologia descrita por Mensor et al. (2001).

\section{RESULTADOS E DISCUSSÃO}

A Tabela 1 apresenta os teores de polifenóis totais e antocianinas monoméricas do extrato supercrítico (SC) e das microcápsulas de jabuticaba (Plinia trunciflora). 
Tabela 1 - Polifenóis totais e antocianinas monoméricas do extrato supercrítico (SC) e das microcápsulas de jabuticaba

\begin{tabular}{lll}
\hline & Polifenóis totais & $\begin{array}{l}\text { Antocianinas } \\
\text { monoméricas }\end{array}$ \\
\hline Extrato SC & $1484,25 \pm 34,21^{\mathrm{a}}$ & $243,47 \pm 3,01^{\mathrm{b}}$ \\
Microcápsulas & $1468,63 \pm 22,90^{\mathrm{a}}$ & $242,54 \pm 3,52^{\mathrm{b}}$
\end{tabular}

Médias seguidas da mesma letra nas colunas não diferem entre si pelo teste de Tukey $(\mathrm{p}<0,05)$.

Nas condições de extração testadas (200 bar, $50{ }^{\circ} \mathrm{C}$ e 2 horas), o extrato supercrítico de Plinia trunciflora apresentou um teor de polifenóis totais de 1484,25 \pm 34,21 mg EAG/100 g de extrato seco e antocianinas monoméricas $243,47 \pm 3,01 \mathrm{mg} / 100 \mathrm{~g}$.

A extração supercrítica com dióxido de carbono da Myrciaria cauliflora, outra espécie de jabuticaba, apresentou teores de polifenóis e antocianinas monoméricas semelhantes aos teores obtidos no presente trabalho para a $P$. trunciflora, sendo que as melhores condições de pressão e temperatura para a extração dos compostos bioativos foram 50 bar e $120{ }^{\circ} \mathrm{C}$ (SANTOS et al., 2011; 2012).

Os teores de polifenóis totais e antocianinas monoméricas presentes no extrato bruto e nas microcápsulas não diferiram significativamente $(\mathrm{p}>0,05)$. O cálculo levou em consideração que as microcápsulas continham teoricamente $25 \%$ de extrato bruto, devido à presença do material encapsulante, a maltodextrina. $\mathrm{O}$ percentual de atividade antioxidante medido através da captura do radical DPPH $(85,11 \pm 0,61 \%)$, também não diferiu, uma vez que, parece estar diretamente relacionado ao teor de polifenóis totais, como também proposto por Jacobo-Velázquez e Cisneros-Zevallos (2009).

Uma forma usual de expressar os valores de atividade antioxidante do ensaio DPPH é calcular a quantidade de antioxidante capaz de sequestrar metade do radical livre presente na solução. Esse índice denomina-se EC50. Quanto menor o valor de EC50 apresentado pelo extrato, maior será sua atividade antioxidante. A concentração do extrato SC de jabuticaba para decrescer a concentração inicial de DPPH em $50 \%$, foi de 78,24 $\pm 0,11 \mu \mathrm{g} / \mathrm{mL}$ valor inferior ao encontrado em extratos de cupuaçu $($ EC $50=177,0 \pm 12 \mu \mathrm{g} / \mathrm{mL})$ e manga $($ EC $50=145,0 \pm 20$ $\mu \mathrm{g} / \mathrm{mL}$ ) (EINBOND et al., 2004).

Segundo Dziezak (1988), apesar de o spray dryer utilizar temperaturas elevadas na secagem, a alta relação superfície/volume das gotículas do produto faz com que a secagem seja quase instantânea, e o fluxo de ar operando na câmara faz com que o tempo de residência das gotículas seja muito pequeno, na ordem de 1 a 2 segundos. Deste modo, a ocorrência de dano térmico é reduzida.

As Figuras 1 e 2 apresentam as microcápsulas de jabuticaba obtidas ao final do processo de atomização e vistas ao microscópio óptico. 


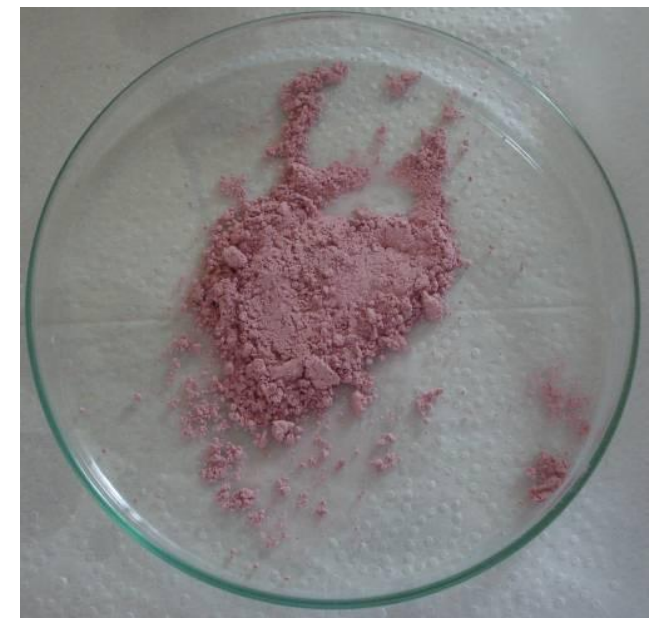

Figura 1 - Microcápsulas de jabuticaba (Plinia trunciflora).

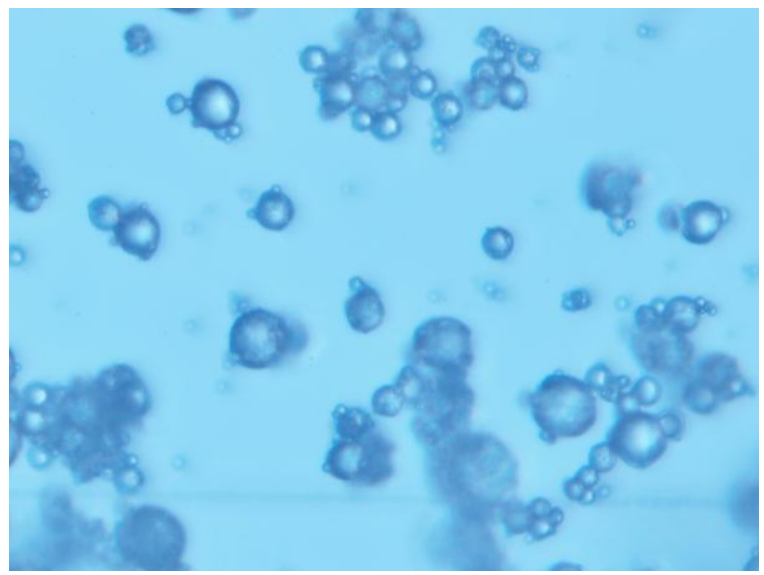

Figura 2 - Microcápsulas de jabuticaba (Plinia trunciflora) vistas ao microscópio óptico (Olympus CX31RBSFA) com aumento de 1000 vezes.

Observando a morfologia das microcápsulas (Figura 2), verifica-se que as mesmas apresentam estrutura esférica, uniforme e sem deformações aparentes em sua estrutura, indicando que as condições de operação, tais como a temperatura de secagem foi adequada. No entanto, houve uma variabilidade quanto ao tamanho das microestruturas. De fato, a variação no tamanho das microcápsulas é comum quando se emprega a atomização por spray dryer (Landim, 2008). Essas diferenças no tamanho das partículas após a atomização podem estar relacionadas principalmente com o fluxo da solução de alimentação. Os estudos morfológicos das partículas depois da etapa de atomização proporcionam uma valiosa informação sobre os fundamentos da secagem das gotas, assim como os fatores físicos e químicos que governam a estrutura da partícula (GHARSALLAOUI et al., 2007). 


\section{CONCLUSÃO}

A atomização por spray dryer mostrou-se vantajosa para a produção de microcápsulas de jabuticaba, uma vez que, não ocasionou perdas dos compostos bioativos e consequentemente da atividade antioxidante das microestruturas ao final do processo.

\section{REFERÊNCIAS}

BRITO, E. S.; ARAúJO, M. C. P.; ALVES, R. E.; CARKEET, C.; ClEVIDENCE, B. A. \& NOVOTNY, J. A. Anthocyanins present in selected tropical fruits: acerola, jambolão, jussara, and guajiru. Journal of Agricultural and Food Chemistry, 55, 9389-9394, 2007.

DAI, J.; GUPTE, A.; GATES, L.; MUMPER, R.J. A comprehensive study of anthocyanincontaining extracts from selected blackberry cultivars: Extraction methods, stability, anticancer properties and mechanisms. Food and Chem. Toxicology. 47. 837-847, 2009.

DESOBRY, S.A.; NETTO, F.M.; LABUZA, T.P. Comparison of spray-drying, drum drying and freezedrying for -carotene encapsulation and preservation. J. Food Science., v.62, n.6, p.1158$1162,1997$.

DUBEY, R.; SHAMI, T.C.; BHASKER RAO, K.U. Microencapsulation Technology and Application. Defence Science. J. 59, 82-95, 2009.

DZIEZAK, J. D. Microencapsulation and encapsulated ingredients. Food Technology, Chicago, v. 42, n. 4, p. 136-151, 1988.

EINBOND, S.L.; REYNERTSON, X.L.; MARGARET, J.B.; EDWARD J.K.; Anthocyanin antioxidants from edible fruits. Food Chemistry. 84 23-28, 2004.

GHARSALLOUI, Adem et al. Applications of spray-drying in microencapsulation of food ingredients: An overview. Food Res. International 40. 1107-1121, 2007.

GIUSTI, M. M.; WALLACE, T. C. Flavonoids as natural pigments. In T. Bechtold, \& R. Mussak (Eds.), Handbook of natural colorants (pp. 261-268). Chichester: John Wiley \& Sons, 2009.

GIUSTI, M.M.; WROLSTAD,R.E. Unit. F1.2.1-13. Anthocyanins. Characterization and measurement with UV-Visible spectroscopy. In: Current Protocols in Food Anal. Chem. Jonh Wiley \& Sons: New York, 2001. 
JACOBO-VELÁZQUEZ, D. A.; CISNEROS-ZEVALLOS, L. Correlations of antioxidant activity against phenolic content revisited: a new approach in data analysis for food and medicinal plants. J. of Food Science, Hoboken, v. 74, n. 9, p. r107-r113, 2009.

LANDIM, E.M.C. Obtenção, caracterização e avaliação da estabilidade de pigmentos naturais microencapsulados. Universidade Federal do Ceará, 2008.

LEITE-LEGATTI, A.V.; BATISTA, A.G.; DRAGANO, N.R.V.; MARQUES, A.C.; MALTA, L.G.; RICCIO, M.F., EBERLIN, M.N.; MACHADO, A.R.T.; DE CARVALHO-SILVA, L.B.; RUIZ, A.L.T.G.; DE CARVALHO, J.E.; PASTORE, G.M.; JUNIOR, M.R.M. Jaboticaba peel: Antioxidant compounds, antiproliferative and antimutagenic activities, Food Research International, 07.044, 2012.

LIN, M.; TSAI, M.; WEN K. Journal of Chromatography A. 830 (2), 387, 1999.

MELO, E.A.; GUERRA, N.B. Ação antioxidante de compostos fenólicos naturalmente presentes em alimentos. Bol. SBCTA. Campinas: v.36, n. 1, p. 1-11, 2002.

MENSOR, L.L.; MENEZES, F.S.; LEITÃO, G.G.; REIS, A.S.; DOS SANTOS, T.C.; COUBE, C.S. AND LEITÃO, S.G. Screening of Brazilian plant extracts for antioxidant activity by the use of DPPH free radical method. Phytother. Res. 15, pp. 127-130, 2001.

NAHAR, L.; SARKER, S.D. Supercritical fluid extraction. In.: SARKER, S.D.; LATIF, Z.; GRAY, A.I. (Eds.). Natural Products Isolation. Methods in Biotechnology. 2. ed. New Jersey: Human press, 2006.

REYNERTSON, K.A.; BASILE, M.J.; KENNELLY, E.J. antioxidant potential of seven myrtaceous fruits. Ethnobotany Res. \& Applications, v. 3, p. 25-35, 2005.

REYNERTSON, K.A.; YANG, H.; JIANG, B.; BASILE, M.J.; KENNELLY, M.E.J. Quantitative analysis of antiradical phenolic constituents from fourteen edible Myrtaceae fruits. Food Chemistry, v.109, p. 883-890, 2008.

SANTOS, D.T.; VEGGI, P.C.; MEIRELES, A. Optimization and economic evaluation of pressurized liquid extraction of phenolic compounds from jabuticaba skins. J. of Food Engineering 108. 444-452, 2012.

SANTOS, D.T.; MEIRELES, A .Optimization of bioactive compounds extraction from jabuticaba (Myrciaria cauliflora) skins assisted by high pressure $\mathrm{CO}_{2}$. Innovative Food Science and Emerging Technologies. 12. 398-406, 2011.

SHAHIDI, F.; HAN, X. Q. Encapsulation of food ingredients. Critical Review in Food Science and Nutrition, 33, 501-547, 1993. 
SHAHIDI, F.; NACZK, M. Food phenolics: souces, chemistry, effects and applications. 1 ed. Lancaster: Technomic Publishing. Co,Inc. 331p, 1995.

SUAVE, J.; DALl'AGNOL, E. C.; PEZZIN, A. P. T.; SILVA, D. A. K.; MEIER, M. M.; SOLDI, V. LIBECON - Liberação Controlada de Agentes Ativos. Health and Environment .J, v. 7, n. 2, dez, 2006.

TODD, R. D. Microencapsulation and flavour industry. Flavour Industry, London, v. 1, n. 11, p. 768-771, 1970.

WESCHE-EBELING, P.; \& ARGAIZ-JAMET, A. Stabilization mechanisms for anthocyanin: The case for copolymerization reactions. In J. Welti-Chanes, G. V. Barbosa- Cánovas, \& J. M. Aguilera (Eds.), Engineering and food for the 21st century (pp. 141-150). Florida: CRC Press, 2002. 